\title{
Repair avoidance: When faithful informational exchanges just don't matter
}

\author{
Bruno Galantucciab* , Benjamin Langsteina, Eliyahu Spivack ${ }^{a} \&$ Nathaniel Paleya \\ a Yeshiva University \\ ${ }^{\mathrm{b}}$ John Cabot University
}

Common-sense intuition suggests that, when people are engaged in informational exchanges, they communicate so as to be reasonably sure that they perform the exchanges faithfully. Over the years, we have found evidence suggesting that this intuition, which is woven into several influential theories of human communication, may be misleading. We first summarize this evidence and discuss its potential limitations. Then we present a new study that addresses the potential limitations.

A confederate instructed participants to "pick up the skask" from a tray containing six objects and move it to a specific location. Since skask is a non-word invented by us, participants had to ask for clarification to perform the instruction faithfully. In contradiction with the intuition that people pursue faithfulness when engaged in informational exchanges, 29 of the 48 participants we tested performed the instruction without asking for clarification. We identified a possible cause for this behavior, which occurred more frequently when avoiding the clarification was unlikely to entail overt consequences. Other factors such as individual differences and the specifics of the experimental settings, if they played a role at all, they did it to an extent that is unlikely to be comparable to that of the role played by overt consequences. Considered together, our various assessments of the extent to which people engage in faithful informational exchanges converge on a simple conclusion: Communicating faithfully is a substantially demanding task, and people often fail at it. We discuss the implications of this conclusion and speculate on its relevance for understanding the evolutionary past of human communication.

Keywords: Human communication; Conversation; Interpersonal interaction

*Please address correspondence to Bruno Galantucci (bruno.galantucci@gmail.com) 
Let us imagine that, while sitting at a table, three friends engaged in the following exchanges.

2

3

Exchange 1

Alice: Mario, can you please pass me the skask?

Mario: Sure

[On the table there are a number of objects. Mario picks up a carafe of wine and passes it to Alice. In the meantime, Alice has left the room to answer a phone call]

Exchange 2 (Mario's answers are all factually true)

Oscar: Mario, I've never heard the word skask before, where does it come from?

Mario: I don't know, I've never heard it before either

Oscar: Do you know what that word means?

Mario: No, I don't

Oscar: Did I hear correctly that Alice asked you to pass her the skask?

Mario: Yes, that's what I've heard too

Oscar: Did you already know that Alice wanted the carafe of wine?

Mario: No, I didn't

Oscar: Let me understand, you passed the carafe to Alice without knowing what she actually asked you to pass?

Mario: Yes that's what I did

Oscar: Did you think she was being silly or making some kind of joke and went along with it?

Mario: No, I didn't think that

Oscar: Do you suffer from any communication disability or mental illness?

Mario: No, I don't

As Oscar's final question suggests, it is very hard to make sense of Exchange 1 in light of Exchange 2. Perhaps Exchange 1 could be an excerpt from a play of the Theater of the Absurdsomething that purposively defies common sense-but definitely not an exchange likely to originate from ordinary circumstances. This simple intuition is woven, more or less explicitly, into several influential theories of human communication. For convenience, here we focus on two of them into which the intuition is woven rather explicitly. The first is a longstanding theory in linguistics and it is proposed by Hockett (1960). According to Hockett (1960), Exchange 1 is at odds with the basic requirement that senders and receivers of a message must share the same associative ties between the words they utter and the things to which the words refer. In his words:

John says, "Please pass the sugar." Bill passes it. ... Thus there is an associative tie between the word sugar and a certain familiar substance: Bill does not pass the salt. (1960, p. 408).

The second theory is a well-established theory in psychology and is proposed by Clark and Wilkes-Gibbs (1986). According to these authors, Exchange 1 is at odds with the principle that senders and receivers of a message are mutually responsible for getting the actual meaning of the message across. In Clark and Wilkes-Gibbs' (1986) words: 
The participants in a conversation try to establish... the mutual belief that the listeners have understood what the speaker meant in the last utterance to a criterion sufficient for current purposes (p. 33).

We believe that these rather diverse theories lead to similar conclusions concerning Exchange 1 because they share a common underlying assumption. The assumption is that, when people are engaged in informational exchanges, they communicate so as to be reasonably sure that they perform the exchanges faithfully (the Faithfulness Assumption).

At first sight, the Faithfulness Assumption does not appear to deserve much scrutiny as not only is it starkly appealing to intuition but it is also consistent with obvious facts. For instance, the existence of common cultural institutions such as financial markets or courts of law would make little sense if we did not assume that people are fully capable of performing faithful informational exchanges. Of course, people can also engage in acts of communication that do not necessarily entail faithful informational exchanges (henceforth we will refer to these acts as non-informational interactions). For example, they can engage in moments of phatic communion (Malinowski, 1923), that is interactions that focus primarily on the establishment and maintenance of pleasant social relations. However, since the faithful transfer of information is not a critical element of non-informational interactions, their existence is not at odds with the Faithfulness Assumption. Non-informational interactions simply fall outside of the assumption's scope. More problematic for the Faithfulness Assumption is the fact that miscommunication is not only rather common in daily life (Mustajoki, Sherstinova, \& Tuomarla, 2018; Tzanne, 2000) but it also occurs in experimentally controlled exchanges which, much as Exchange 1, seem to fall squarely within the assumption's scope (Keysar, 2007). Could this be an indication that the Faithfulness Assumption might have limitations that go beyond the scope reduction imposed by non-informational interactions? Over the years, we have found evidence consistent with this hypothesis.

We stumbled onto the first piece of evidence while studying people engaged in the task of inventing novel communication systems in the absence of pre-established ones such as speaking or writing (Galantucci, 2005; Galantucci, Fowler, \& Richardson, 2003). People turned out be quite good at the task - a finding consistent with field observations (e.g., Goldin-Meadow \& Feldman, 1977; Kegl, Senghas, \& Coppola, 1999; Sandler, Meir, Padden, \& Aronoff, 2005) - but there were remarkable cases of failure. For the most part, these cases were due the fact that people exhibited important limitations in their basic communicative abilities (Galantucci, 2009; Galantucci \& Roberts, 2012; Galantucci \& Steels, 2008). For instance, some individuals understood communicative acts performed by others - and used them for hours to inform their behavior-but did not reciprocate the acts, even when it was obvious that such reciprocation was necessary to avoid critical communication problems. These individuals did not seem to be even aware of the problems. Other individuals produced identical communicative acts but intended them to convey different meanings depending on information that was accessible only to them (cf. Keysar \& Henly, 2002; Lane \& Ferreira, 2008 for similar phenomena with natural language use). Predictably, the recipients of these cryptic communicative acts repeatedly failed 
to understand them as intended. Yet, when this happened, the individuals who performed the cryptic acts reacted with surprise and disappointment, seemingly oblivious to the fact that their own behavior caused the failures. Considering that all of the individuals described above reported having normal communicative abilities, it became apparent that the challenging task had offered a new opportunity for assessing these abilities (Galantucci \& Roberts, 2012). The findings were a bit surprising. When people could not use pre-established communication systems, not only did they sometimes show important limitations in their ability to communicate effectively, but they also seemed to have little awareness of these limitations. These findings forced us to reconsider our initial assumptions concerning human communicative abilities, suggesting to us the hypothesis that the Faithfulness Assumption might have important limitations. Our first step in testing this hypothesis was to rule out the possibility that the findings from which it originated were artifacts caused by the highly unusual task of inventing a novel communication system. In consequence, we began investigating human communication in conditions much closer to the ordinary ones.

The Faithfulness Assumption has two critical entailments. The first is that people should monitor the faithfulness of the exchanges they perform, detecting any problem that might compromise it. The second is that, when people become aware of any such problem, they should address it. We began our test of the Faithfulness Assumption by focusing on the first entailment, measuring the extent to which people engaged in spontaneous conversations detected the presence of communication problems (Galantucci \& Roberts, 2014; Galantucci, Roberts, \& Langstein, 2018; Roberts, Langstein, \& Galantucci, 2016). The measure relied on a simple method that has been often used to investigate attention in psychology: People are exposed to an oddity in a seemingly mundane event (e.g., a change in interlocutor during a oneto-one conversation; Fenn et al., 2011; Simons \& Levin, 1998) and then they are interviewed to determine whether they noticed the oddity. In our case, the oddity was an obvious communication problem that we introduced in spontaneous conversations.

108

109

110

111

112

113

114

115

116

117

118

119

120

In a first study (Galantucci \& Roberts, 2014), we assigned a simple communication task to two pairs of participants who did not know of each other's existence. In particular, each pair had to converse about a cartoon by sending instant messages from different physical locations. Unbeknownst to the participants, we occasionally crossed their conversations in such a way that each member of a pair ended up conversing with a member of the other pair. In other words, participants conversed multiple times with a stranger, who was involved in a different conversation focused on a different cartoon. The crossings of the conversations lasted 30 seconds, occurred four times over the course of 15 minutes, and the instant messaging platform did not reveal any overt sign of their existence. The logic of the study was straightforward: If people have limited ability to monitor the faithfulness of the messages they exchange, they may not detect the conversational incoherencies caused by the crossings. The prediction was tested in two experiments. In the first, aimed at investigating narrowly-focused conversations, each participant in a pair was told that their partner saw the same picture, colored differently, and 
121 that their task was to find the color differences. Five of the 15 participants for whom the 122 crossings had created obvious conversational incoherencies failed to notice them. In a second 123 experiment, aimed at investigating more broadly focused conversations, participants were told 124 that their task was to discuss which of the celebrities depicted in the cartoon they would most

125 and least like to spend a day with. Three of the 11 participants for whom the crossings had created obvious conversational incoherencies failed to notice them.

A follow-up study (Roberts et al., 2016) replicated the second experiment of the first study with a tighter manipulation of conversational coherence. Again unbeknown to the participants, we swapped two messages in their conversation for messages of our own. These swaps were designed to consistently generate obvious incoherencies. They also allowed us to manipulate the kind of incoherence encountered by the participants. In one experiment, the incoherent message concerned a celebrity who was not in the cartoon and was not mentioned before in the conversation. For example, while Oprah was not in the cartoon and was not mentioned before in the conversation, the manipulated message would read: "Of these six, Oprah is kind of an icon for people like me". 15 out of 40 participants failed to notice the incoherent message. In a second experiment, the incoherent message implied that its sender was of a different gender from the gender expected by the receiver. For example, if the receiver's partner was a man, the manipulated message would read: "Hillary Clinton is an icon for women like me" (Hillary Clinton was one of the celebrities depicted in the cartoon). Participants met their partners at the start of the experiment and were in no doubt as to who they would be conversing with. Yet, 11 out of 33 participants failed to notice the incoherent message. In sum, people engaged in instant messaging conversations showed important limitations in detecting obvious communication problems.

Our next step was to rule out the possibility that this finding was specific to instant messaging, an artificial medium that is clearly different from the natural medium of human communication ${ }^{1}$. We engaged 30 participants in one-to-one spontaneous conversations with a lab confederate (Galantucci, Roberts, \& Langstein, 2018). The conversations occurred face-toface and were prompted by the task of jointly ranking in order of humorousness and oddness five "would you rather" questions (e.g., "would you rather live on a tree or in a cave?"). After about 8 minutes of mundane exchanges, the confederate uttered the sentence "colorless green ideas sleep furiously". The sentence is not only bound to cause a communication problem in almost any imaginable conversational context, but is also devoid of meaning. Yet 20 of the 30 participants - when interviewed soon after the confederate uttered the sentence - believed that no such sentence had been introduced into their conversation. Moreover, of the ten who though that such a sentence had been introduced, only one could correctly recognize it in a list that included 20 other nonsensical sentences. In other words, people's capability to detect

\footnotetext{
${ }^{1}$ For example, instant messaging lacks two critical features of face-to-face conversation: the copresence of the conversants in the same physical space and their mutual visibility (Clark \& Brennan, 1991).
} 
communication problems might be even lower in face-to-face conversations than in instant messaging exchanges.

Taken together, the three studies provide converging evidence consistent with the conclusion that people's sensitivity to communication problems has important limitations. These limitations are in stark contrast with the Faithfulness Assumption. However, as pointed out by Micklos, Walker, and Fay (2020), this conclusion might be undermined by two potential confounds. On the one hand, the limitations we observed might have been caused by the fact that people encountered the communication problems while they were engaged in moments of non-informational interaction. With the exception of one experiment, in fact, all of our experiments involved spontaneous conversations aimed at performing tasks with low informational demands (the exception is the first experiment of the 2014 study). During these conversations, moments of non-informational interaction were a definite possibility. On the other hand, the limitations we observed might have been caused by memory failures. In particular, it is possible that the participants of our studies noticed the communication problems when they encountered them but then, when we interviewed them after the encounters, they failed to remember that they had noticed. While we believe that these potential confounds are unlikely to explain all of the 54 cases in which participants did not report having noticed the communication problems, we agree with Micklos and colleagues that a more stringent assessment of the Faithfulness Assumption requires ruling them out.

In the current study, we ruled out the possibility of non-informational interactions by inserting communication problems in highly structured exchanges that, much as Exchange 1, were not only informational in nature but also led to noticeable visible consequences. As for the possibility of memory failures, we ruled them out by observing people's immediate reactions to a communication problem they encountered. However, rather than focusing on whether people noticed the problem, we focused on whether they attempted to resolve it. We made this choice for three reasons. First, because it still allowed us to test a critical entailment of the Faithfulness Assumption. As we noted above, in fact, if people noticed communication problems but did not resolve them, the faithfulness of their informational exchanges would still be in jeopardy. Second, because the choice allowed us to circumvent the intricacies of measuring noticing, a response which can be difficult to sharply define (Nisbett \& Wilson, 1977). Detecting whether people attempt to resolve a problem is a simpler endeavor. Third, because the choice provided an opportunity to test a hypothesis originating from some unpublished findings of the face-toface study discussed above (Galantucci et al., 2018). Although we did not design that study to assess participants' immediate reactions, we had video footage of some of the trials in which the sentence "colorless green ideas sleep furiously" was uttered. According to the Faithfulness Assumption, one would expect the footage to document participants' attempts to address the communication problem, performing what is known as an other-initiated-repair (Schegloff, 2000; henceforth repair, for simplicity). The footage indeed contained a few repairs (e.g., Exchange $A$ in Box 1, from Clip $A$ in the Appendix), but also several unexpected responses such 
as laughing (e.g., Exchange B, from Clip B), completely ignoring the sentence (e.g., Exchange $C$, from Clip C), or even assenting to it (e.g., Exchange D, from Clip D).

$\begin{array}{ll}\text { Exchange A } & \text { Confederate: colorless green ideas sleep furiously } \\ & \text { Participant A: what? } \\ \text { Exchange B } & \text { Confederate: colorless green ideas sleep furiously } \\ \text { Exchange C } & \text { Participant B: [laughs]... all right... so we are at } \\ & \text { Confederate: colorless green ideas sleep furiously } \\ \text { Exchange D } & \text { Participant C: you never played would you rather? } \\ & \text { Confederate: colorless green ideas sleep furiously } \\ & \text { Participant D: yeah }\end{array}$

Box 1. Exchanges between the participants and the confederate

Why did participants not repair such a conspicuously problematic sentence? Inspection of the footage suggested that, for some of them, it was possible that they were so focused on the flow of the conversation that they did not even process the sentence. This seemed to be the case for Participants C and D in Box 1, who both reported not noticing the communication problem when interviewed at the end of the conversation. These participants seemed to exhibit a form of attentional deficit (cf. Fenn et al., 2011; Simons \& Levin, 1998), which we labeled content deafness (Galantucci et al., 2018). In the case of other participants, however, this explanation was rather implausible. This seemed to be the case for Participant B in Box 1, for example, who indeed reported noticing the communication problem when interviewed at the end of the conversation. We hypothesize that participants like Participant B avoided the repair because the communication problem they encountered was unlikely to lead to overt consequences (the Overt Consequences Hypothesis). The participant and the confederate, in fact, were engaged in a task that they could adequately perform even if they ignored a momentary communication problem. In particular, as mentioned above, they jointly ranked in order of humorousness and oddness five "would you rather" questions. This open-ended task led to rather casual conversations during which moments of non-informational interaction could have made irrelevant the potential consequences of a communication problem. As we will describe below, in the current study we tested the Overt Consequences Hypothesis through an experimental manipulation.

\section{Study}

A confederate instructed participants to move ordinary objects from a tray (see Figure 1A for an example) to a specific location on a chessboard. After a few mundane instructions, the 
confederate asked the participants to "pick up the skask and move it to C5". Since "skask" is a nonword created by us, the instruction contained a communication problem that was not only likely to be noticed but which also created a clear need for a repair. The repair was indeed critical for the faithfulness of the informational exchange: If participants avoided it, they might move a different object than the one intended by the confederate. To ascertain whether participants performed a repair, we recorded their immediate reactions to the problem. A systematic inspection of these recordings provided an assessment of the Faithfulness Assumption that not only did not rely on participants' memory but it also originated from conversations that were unlikely to lead to non-informational interactions. If the hypothesis that the Faithfulness Assumption has important limitations is correct, the inspection should reveal a number of cases in which participants do not initiate a repair in reaction to the communication problem.

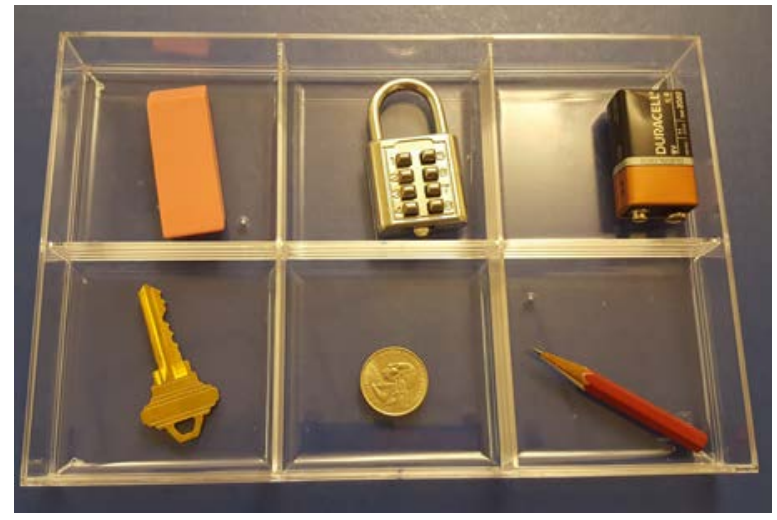

(A)

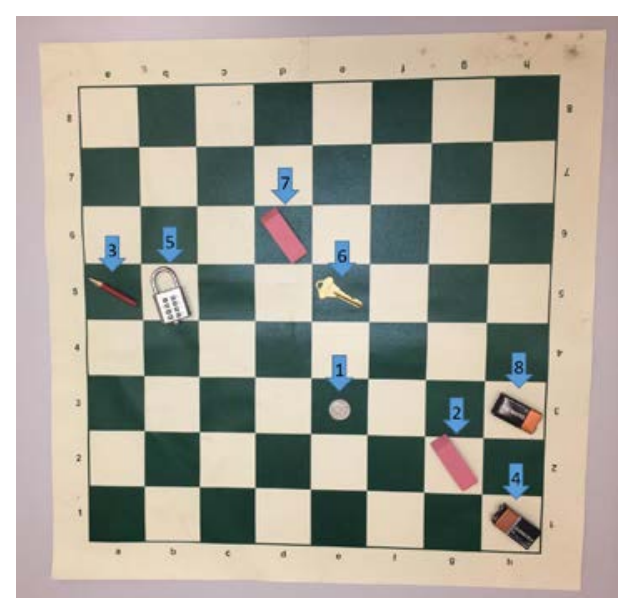

(B)

Figure 1. (A) The tray used for the first mundane round of the game. (B) The picture illustrating where each object was to be placed and in what order the instructions were to be given in the first mundane round.

To test the Overt Consequences Hypothesis, we manipulated the probability that avoiding a repair could lead to overt consequences. In particular, two people-the confederate and an experimenter - had a clear view of the chessboard onto which the participant moved the objects. Furthermore, we informed participants that the session could be video recorded. In other words, if participants moved a different object than the one intended by the confederate, it was clear to them that someone was likely to notice the mistake. In the High Probability Consequences condition (henceforth HPC condition), four of the six objects on the tray had a well-known name while the other two did not (see Fig. 2A below). In consequence, avoiding a repair of the problematic instruction entailed a $50 \%$ probability of making a noticeable mistake. In the Low Probability Consequences condition (henceforth LPC condition), five of the six objects 
on the tray had a well-known name while the sixth did not (see Fig. 2B below). In consequence, when participants were asked to "pick up the skask and move it to C5", they could avoid a repair while, reasoning by exclusion (cf. Golinkoff, Hirsh-Pasek, Bailey, \& Wenger, 1992), they can still expect a rather low probability of making a noticeable mistake. Note that the probability is low but not null. The confederate, in fact, might have intended to refer to one of the objects with a well-known name and the instruction heard by the participant might have been the result of a communication problem. If the Overt Consequences Hypothesis is correct, participants in the HPC condition should be more likely to perform a repair than participants in the LPC condition.

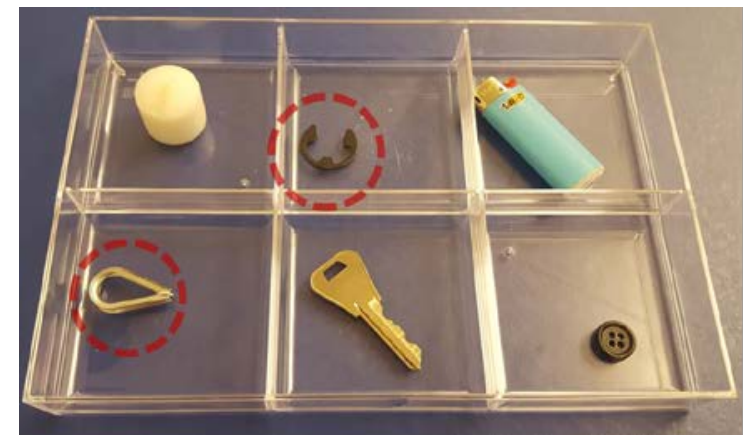

(A)

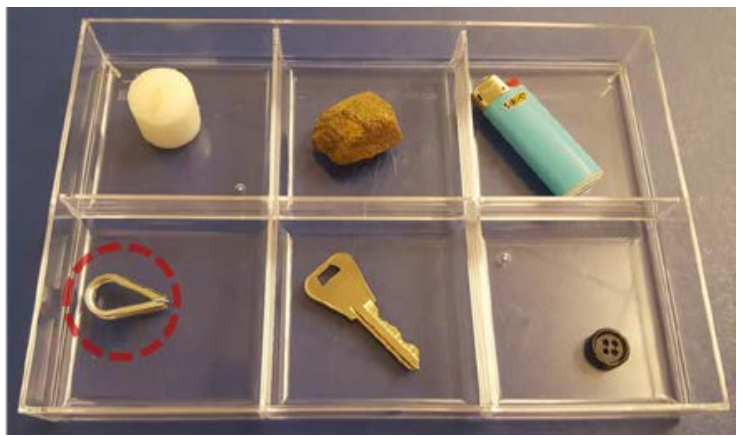

(B)

Figure 2. (A) The six objects of the HPC condition. The two objects without a well-known name are circled in red (at the hardware store where they were purchased, the object on the bottom row was sold as "wire rope thimble" while the object on the top row was sold as "retaining ring"); (B) The six objects of the LPC condition. The object without a wellknown name is circled in red (for half of the participants it was the other object without a well-known name from Figure 2A).

\section{Method}

\section{Participants}

48 English-speaking students from New York City, with no deficits in communicative ability, were recruited through online advertisements. Participation was compensated with $\$ 25$. The average age of the participants was 26.73 years $(S D=8.21)$ and there were 15 female participants, 31 male participants, and 2 participants who preferred not to declare gender.

\section{Ethical statement}

Ethical approval was granted by the Institutional Review Board at Yeshiva University. All participants gave written consent to participate by signing a consent form. The consent form included a paragraph stating that any behavior that took place during the session could be video recorded. 


\section{Procedure}

Upon arriving at the lab, the participant and the confederate (one of us, NP) were introduced to one another as two participants who had been randomly paired up. The confederate's age (24 years) was similar to the average age of the participants and he was dressed casually. For the entire duration of the session, the confederate acted so as to minimize the likelihood that the participant would perceive his association with the lab, and the experimenter (one of us, ES) interacted with the confederate in the same manner as with the participant. For example, the experimenter asked the confederate for his name in the presence of the participant, as if they had never met before. For this reason, in the remaining part of this section we will refer to the confederate and the participant as "the participants" and make a distinction between them only when it is critical for the design of the study. After the participants reviewed and signed the consent form for the study, the experimenter escorted them into a room. He then told them that the session involved a number of tasks, without specifying how many. The first task was a Cover Task.

\section{Cover Task}

In order to dissimulate the goal of the study, we began the session with a Cover Task. In particular, the experimenter asked the participants to rank, in order of difficulty and humorousness, four "would you rather" questions taken from the same set of questions used for the 2018 study described above (Galantucci, Roberts, \& Langstein, 2018). Participants were instructed to perform the Cover Task in one of two ways. Half of them (12 in the HPC condition and 12 in the LPC condition) were instructed to perform it individually with pen and paper, while being seated so that they faced opposite directions. For these participants, there was essentially no chance of engaging in casual conversations. In what follows, we will refer to this task as the "Formal Settings Cover Task". The other half of the participants (12 in LPC condition and 12 in the HPC condition), performed the Cover Task jointly rather than individually. In particular, similarly to the 2018 study (Galantucci, Roberts, \& Langstein, 2018), the experimenter instructed the participants to negotiate, through face-to-face conversations, a joint ranking of the four "would you rather" questions. As we learned in the 2018 study, this task elicits lively conversations that consist entirely of spontaneous exchanges. In what follows, we will refer to this task as the "Informal Settings Cover Task". We used the two different cover tasks to assess the extent to which the formality of the settings affected participants.

For both cover tasks, the experimenter collected the rankings after five minutes had elapsed. He then asked each participant to answer in writing the following questions:

Question 1: On a scale from 0 to 10, how good of a job did you do in ranking the questions? Question 2: On a scale from 0 to 10, how good of a time did you have with your partner on this first task?

Question 3: On a scale from 0 to 10, how good do you feel right now? 
We asked these questions for two reasons. First, to further dissimulate the goal of the study, decoying participants who speculated about it into a very wide range of possibilities. The second reason was that of assessing the extent to which the difference between the two cover tasks affected participants. Once participants had answered the three questions, the experimenter introduced a second task, the study's Critical Task.

\section{Critical Task}

The experimenter asked the participants to sit across from each other at a round table (diameter $107 \mathrm{~cm})$ and placed between them a large chess board $(61 \mathrm{~cm} \times 61 \mathrm{~cm})$ with numbers and letters demarcating rows and columns. He told participants that they would be playing a simple game in which one of them would play the role of a director and the other the role of a mover. He then asked the participant to blindly pick from a box one of two pieces of paper to determine which role s/he would play. Unbeknownst to the participant, both pieces were labeled mover. In consequence, the participant always played the role of mover and the confederate always played the role of director. The experimenter told participants that, for each round of the game, the director would give instructions to the mover on how to move objects from a tray (see Figure 1A for an example) onto the chessboard and then back into the tray. In order to explain how the director was to know which instructions to give in each round, the experimenter explained that he would give the director a picture of the chessboard illustrating where each object was to be placed and in what order the instructions were to be given (Figure 1B). After briefly showing the picture to the participants, the experimenter instructed the confederate to request a move by saying "please pick up the OBJECT and move it to COORDINATE" (e.g., "please pick up the candle and move it to C3") and, once the object had been placed on the board, to say "move it back". We adopted this procedure to maximize the likelihood that the only portions of the instructions that could lead to communication problems were the portions referring to the object and the coordinate.

Participants played four rounds of the game. The first two and the fourth rounds were mundane rounds in which the participant moved objects with well-known names (see Figure 1 for an example). The third round contained the critical instruction "please pick up the skask and move it to C5" (henceforth critical instruction). As described in the introduction, the 24 participants we randomly assigned to the HPC condition received the critical instruction while moving objects from the tray in Figure 2A and the 24 participants we randomly assigned to the LPC condition received it while moving objects from the tray in Figure 2B. In the HPC condition, the critical instructions occurred after four instructions involving each of the four objects with well-known names (e.g., "please pick up the lighter and move it to F2") and was followed by two more mundane instructions. In the LPC condition, the critical instructions occurred after five instructions involving each of the five objects with well-known names and was followed by one more mundane instruction. In other words, participants in this condition 
could easily infer the likely meaning of the critical instruction. In consequence, while they might have still felt the need to initiate a repair to make sure they heard the instruction correctly, avoiding the repair was unlikely to lead to overt consequences. We adopted this procedure to maximize the contrast between the two conditions.

\section{Questionnaire}

After the completion of the Critical Task, the experimenter told the participant and the confederate that he would escort them to different rooms to answer a questionnaire. The questionnaire, which in reality was administered only to the participant, included two main sections.

Individual differences questionnaire. In the first section we collected information about individual differences among the participants. This section included standard demographic information such as age and gender. It also included an inventory to measure the Big Five personality dimensions (BFI-2-S; Soto \& John, 2017), a measure of social intelligence (the Mind in the Eye Test; Baron-Cohen, 2001), and a measure of multilingualism (the LEAP-Q inventory; Blumenfeld \& Kaushanskaya, 2007). We collected this information because we wanted to know whether it would enable us to predict the likelihood that participants performed a repair after receiving the critical instruction.

Session questionnaire. In the second section of the questionnaire we collected information about the session. The section included the following questions:

Question A: What do you think was the purpose of the study?

Question B: Do you think that your partner was acting genuinely?

Question C: Do you remember hearing the word "skask" during the experiment?

Question D: (asked only if answer to Question C was "yes"): Do you know what "skask" means? Question E: (asked only if answer to Question D was "yes"): What does "skask" mean? Question F: (asked only if answer to Question D was "no"): Did you let your partner know that you didn't know the meaning of the word "skask"?

Question G: (asked only if answer to Question F was "no"): Which of the options below best accounts for why you didn't say anything when your partner used the word "skask"?

- I thought that asking for clarification would disrupt the flow of the conversation.

- I thought that it was clear enough which item my partner was referring to.

- $\quad$ Other (please specify)

Questions A-B provided a check of the fact that our cover procedures worked as intended, Questions C-G provided insight into participants' subjective experiences of the critical instruction. 


\section{$377 \quad$ Results}

378 Experimental settings check. As revealed by their answers to Question A, none of the

379 participants correctly guessed the purpose of the study. However, as revealed by Question B,

380 four participants correctly guessed that the confederate was not acting genuinely. In

381 consequence, the data from these participants were not used for the analyses that follow.

382 An inspection of the video recordings revealed that participants made virtually no mistakes in

383 moving the correct objects (as well as in placing the objects in the correct locations on the

384 chessboard). This suggests that our experimental settings worked as intended in making

385 participants aware that their mistakes could be noticed.

386 An analysis of the answers to Questions 1-3 (the three questions we asked participants at the 387 end of the cover task), revealed that the difference in cover task clearly affected participants' 388 answers to Question 2 ("On a scale from 0 to 10, how good of a time did you have with your 389 partner on this first task?"). In particular, the answers of the participants who performed the 390 Formal Settings Cover Task $(\mathrm{Mdn}=5, \mathrm{IQR}=1.5-7)$ were significantly lower than the answers of 391 the participants who performed the Informal Settings Cover Task (Mdn = 8, IQR = 7-10), U = $39277.5, \mathrm{p}<001, \mathrm{r}=.59$. As for Questions 1 and 3, the answers of the participants who performed the 393 Formal Settings Cover Task (Question 1: $\mathrm{Mdn}=8, \mathrm{IQR}=6.5-10$; Question 3: $\mathrm{Mdn}=7$, IQR = 5-9) 394 were also slightly different than the answers of the participants who performed the Informal 395 Settings Cover Task (Question 1: $\mathrm{Mdn}=8, \mathrm{IQR}=7-10$; Question 3: $\mathrm{Mdn}=8, \mathrm{IQR}=7-9$ ). These 396 differences, however, were not significant. Before describing the impact of the difference in 397 cover task on the occurrence of repair after the critical instruction, we will first describe the 398 procedure we used to detect such occurrences.

399 Detecting the occurrence of a repair after the critical instruction

400 Two of us (ES and NP) independently inspected the recordings of the critical instruction in 401 order to detect whether participants performed a repair. The criterion used for the detection 402 was that participants were to be considered as not having performed a repair only if they 403 performed the critical instruction without requesting any clarification from the confederate. In 404 case of disagreement, the participant was to be considered as having performed a repair. There 405 were no disagreements. The video clips for all of the participants who were considered as not 406 having performed a repair can be viewed through the URL links in the Appendix. In addition, 407 Clips 7 and 22 provide examples of participants who were considered as having performed a 408 repair. 
410 For convenience, in Table 1 we provide a synopsis of the repair occurrences. As reported in the 411 table, 15 of the 44 total participants (34.1\%) performed a repair after the critical instruction and $41229(65.9 \%)$ did not (henceforth we will refer to these participants as repair avoiders).

\begin{tabular}{|c|c|c|c|c|}
\hline \multirow{3}{*}{$\begin{array}{c}\text { Low } \\
\text { Formality } \\
\text { Cover Task }\end{array}$} & & $L P C$ & HPC & Total \\
\hline & Repair & $1(9.1 \%)$ & $4(40 \%)$ & $5(23.8 \%)$ \\
\hline & No repair & $10(90.9 \%)$ & $6(60 \%)$ & 16 (76.2\%) \\
\hline \multirow{3}{*}{$\begin{array}{c}\text { High } \\
\text { Formality } \\
\text { Cover Task }\end{array}$} & Total & 11 & 10 & 21 \\
\hline & Repair & $2(18.2 \%)$ & $8(66.7 \%)$ & $10(43.5 \%)$ \\
\hline & No repair & $9(81.8 \%)$ & $4(33.3 \%)$ & $13(56.5 \%)$ \\
\hline \multirow{4}{*}{$\begin{array}{l}\text { Across cover } \\
\text { tasks }\end{array}$} & Total & 11 & 12 & 23 \\
\hline & Repair & $3(13.6 \%)$ & $12(54.5 \%)$ & $15(34.1 \%)$ \\
\hline & No repair & $19(86.4 \%)$ & $10(45.5 \%)$ & $29(65.9 \%)$ \\
\hline & Total & 22 & 22 & 44 \\
\hline
\end{tabular}

Table 1. Breakdown of repair occurrences.

413 Overt Consequence Hypothesis. In the HPC condition, 12 participants (54.5\%) performed a 414 repair after the critical instruction and 10 (45.5\%) did not. In the LPC condition, three

415 participants (13.6\%) performed a repair after the critical instruction - confirming our intuition 416 that even in this condition a repair was not entirely superfluous - and 19 participants (86.4\%)

417 did not. The difference in repair occurrences between the two conditions is statistically

418 significant $(\mathrm{p}=.005 \text {, one-tailed Fisher's exact test, Cramer's V }=.432)^{2}$.

419 Formality of settings. Five of the participants who performed the Formal Settings Cover Task 420 (23.8\%) performed a repair after the critical instruction and 16 (76.2\%) did not. Ten of the 421 participants who performed the Informal Settings Cover Task (43.5\%) performed a repair after 422 the critical instruction and 13 participants (56.5\%) did not. The difference in repair occurrences

2 The difference remains significant if the four excluded participants are included $(p=.003$, one-tailed Fisher's exact test, Cramer's V = .43). 
423 between the two cover tasks is not statistically significant ( $p=.213$, two-tailed Fisher's exact test,

424 Cramer's V =.207) 3 .

425 Individual differences. A binary logistic regression revealed that none of the individual

426 differences we collected could reliably predict the occurrence of repair [all ps > .12; omnibus test

427 of full model: $\left.\mathrm{X}^{2}(9, \mathrm{~N}=44)=3.8, \mathrm{p}=.91\right]^{4}$.

\section{Discussion and conclusions}

429 The current study provided a clear result: Not only did participants frequently avoid repair 430 after encountering a non-word that they had never heard before but also ten of them did so 431 even when, as in the HPC condition, this was likely to jeopardize the faithfulness of an 432 informational exchange. Considering that there were no non-informational interactions during 433 the Critical Task, the behavior of these ten participants represents the starkest violation the

434 Faithfulness Assumption we have ever documented: People engaged in informational 435 interactions often forego the repair of a critical communication problem. Is it possible that, as 436 suggested by our previous studies, the ten repair-avoiders in the HPC condition did not even 437 detect the communication problem? This possibility seems to be inconsistent with the fact that 438 none of the seven repair-avoiders in the HPC who were asked Questions G answered it by 439 choosing the option "I thought that it was clear enough which item my partner was referring to". In 440 any event, to more soundly rule out the possibility of a failure in detecting the communication 441 problem, we measured the time elapsed between the moment an instruction began (the 442 utterance of the word "Please") and the moment in which the participant touched an object in 443 the tray (the Pick-up Time) ${ }^{5}$. If the repair-avoiders did realize that they had encountered a 444 communication problem, then the Pick-up Times for the critical instruction should be longer 445 than for the instructions that immediately preceded it. Figure 3B illustrates the Pick-up Times 446 for the critical instruction and the three preceding instructions. As is apparent in the figure, the 447 Pick-up Times for the critical instruction were markedly longer than the Pick-up Times for each 448 of the three preceding instructions. Indeed, the difference was statistically significant for each of 449 the three pairwise comparisons (all paired-samples t-tests yielded ps $<.05$, with all Cohen's ds > 450 1). For comparison, Figure 3A illustrates the same measurements for 18 of the repairer avoiders 451 in the LPC condition ${ }^{6}$. The measurements suggest much less marked differences in Pick-up 452 Times. Indeed, the difference between the Pick-up Times for the critical instruction and the 453 preceding one was no longer significant, $\mathrm{t}(17)=.18$; Cohen's $\mathrm{d}=.04$, while the other two

\footnotetext{
${ }^{3}$ The difference remains nonsignificant if the four excluded participants are included $(\mathrm{p}=.371$, two-tailed Fisher's exact test, Cramer's V = .172).

${ }^{4}$ The result is similar if the four excluded participants are included [all ps $>.25$; omnibus test of full model: $\mathrm{X}^{2}(9, \mathrm{~N}=48)=3.77, \mathrm{p}=.93$ ]

${ }^{5}$ The measurement could not be performed for one participant because of a technical failure during the recordings.

${ }^{6}$ The measurement could not be performed for one participant because of a technical failure during the recordings.
} 
differences remained significant (both paired samples t-tests yielded $\mathrm{ps}<.05$ ) but were smaller in size (both Cohen's ds <.6). This contrast highlights the struggle faced by the repair-avoiders in the HPC condition. A majority of these ten participants were clearly aware of the struggle. In answer to Questions C- $\mathrm{D}^{7}$, in fact, six of them reported remembering hearing the word "skask" and not knowing what it meant. Sometimes, as in the case of Participant 2, there was little for us to infer, as the struggle was already evident in his overt behavior: He picked up both objects without a well-known name, smiled, and then moved one of the objects (see Clip 2). The four remaining repair-avoiders in the HPC condition reported not remembering hearing the word

\section{LPC condition}

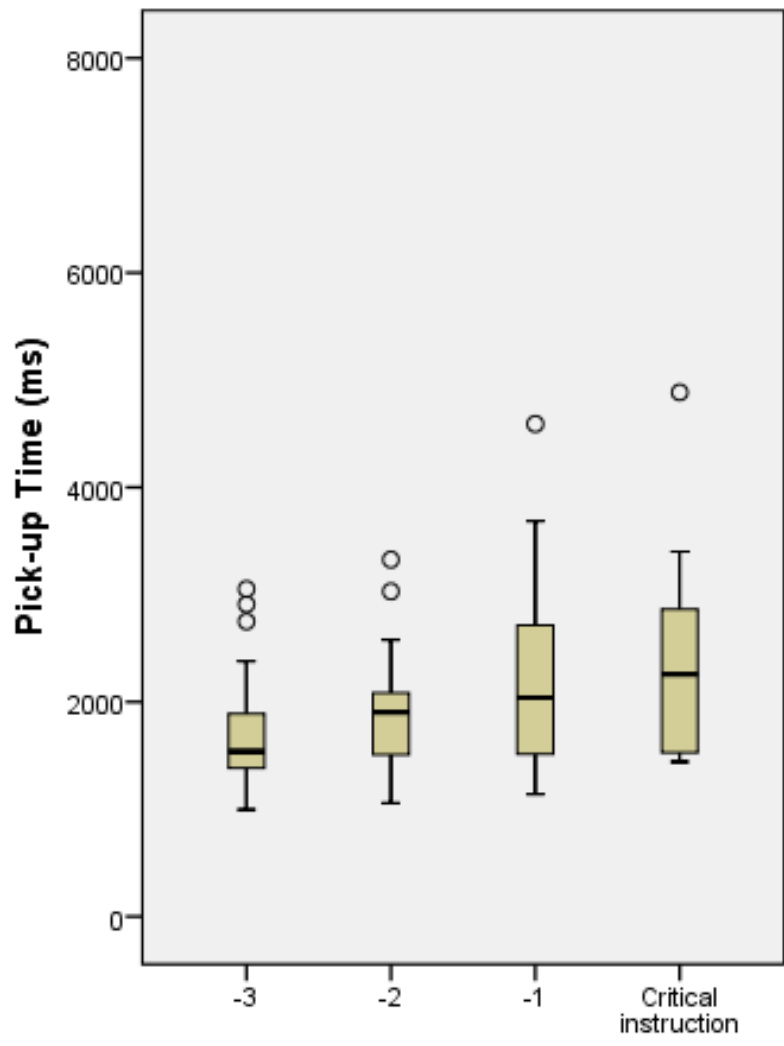

(A)
HPC condition

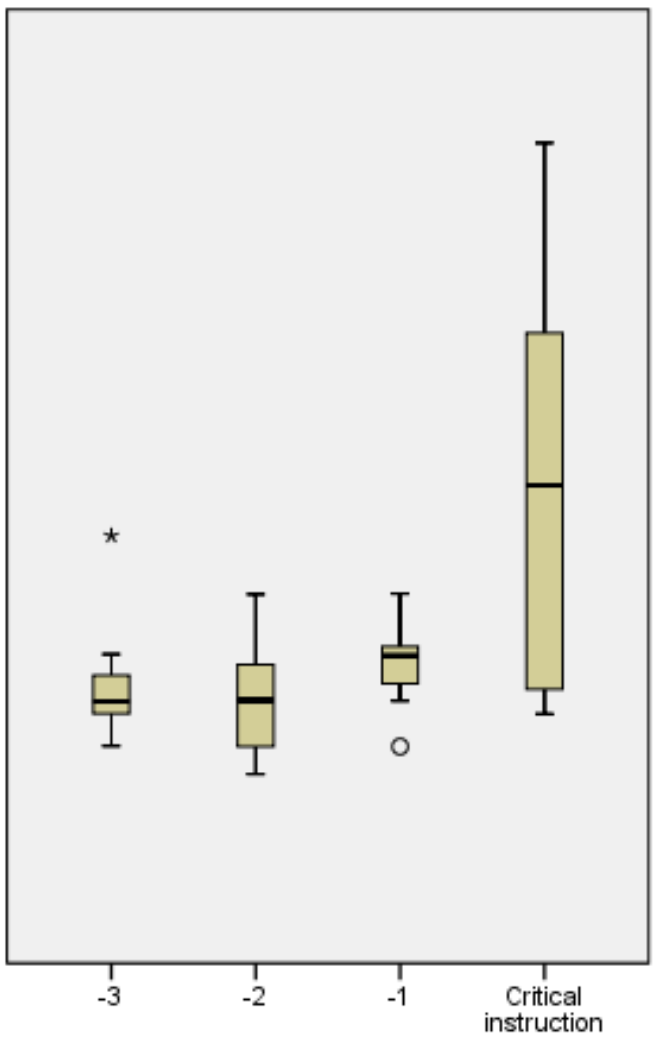

(B)

Figure 3. (A) Boxplots for the Pick-up Times of the critical instruction and the three preceding instructions in the LPC condition; (B) Boxplots for the Pick-up Times of the critical instruction and the three preceding instructions in the HPC condition. (The boxes represent the interquartile ranges, the error bars the ranges, the horizontal line the medians, the circles the outliers, and the asterisks the extremes.)

\footnotetext{
${ }^{7}$ We will discuss the answers to the Questions E-G in the General discussion.
} 
"skask". Considering that also their mean Pick-up Times were longer for the critical instruction than for each of the three preceding instructions, this finding is consistent with the possibility that memory failures might have contributed to the findings of our previous tests of the Faithfulness Assumption (Galantucci et al., 2018; Roberts et al., 2016; Galantucci \& Roberts, 2014).

The current study also offered two insights concerning the reasons why people might avoid clearly needed repairs when engaged in informational exchanges. On the one hand, we found positive evidence in favor of the Overt Consequences Hypothesis: People are more likely to avoid repair when the avoidance is less likely to lead to overt consequences. On the other hand, our results suggest that the effects of individual differences and of the experimental settings on repair avoidance, if existent at all, are unlikely to be comparable in size to the effect of overt consequences. Perhaps larger effects could be found in the future by considering other hypotheses.

For example, people might avoid repairs because they expect relevance from their interlocutors (Sperber \& Wilson, 1986) and patiently wait for a deferred solution of the communication problems they encounter. Participant 2, a repair-avoider in the HPC condition, provides a good example of this possibility. In answer to Question G, in fact, he said that he avoided repair because "I figured a guess was probably good enough and that, given that he had an image of the board, he'd correct me if I picked wrongly".

Also, perhaps out of concerns for face management (Goffman, 1955), people may be reluctant to interrupt the flow of an interaction to let a stranger know that either he said something problematic or they do not know a word of their native language. This explanation seems particularly compelling for the three repair avoiders in the HPC condition who nonetheless spontaneously initiated repairs during the mundane rounds of the Critical Task. For example Participant 6, when asked to "pick up the skask", picked up one of the two objects without a well-known name, without saying a word (see Clip 6). Yet, she initiated a repair a few rounds after the critical instruction, aimed at clarifying the correct location on the chessboard where to place the object she was picking up from the tray (see Clip 6a). In other words, while Participant 6 felt comfortable asking clarifications concerning instructions that she did not fully understand, she did not feel comfortable addressing the odd situation caused by the Critical Instruction. This occurred despite the fact that, as demonstrated by the repair she performed after the critical instruction, she seemed to care about performing the task accurately.

Finally, another possibility is that, as suggested by our previous studies (including those in which people invented new communication systems), the very ability to detect communication problems might be limited. As we noted above, this possibility cannot explain the results of the current study but it may explain other occurrences of repair avoidance such as some of the ones we documented when discussing the footage of the 2018 study (Galantucci, Roberts, Langstein, 
2018). In other words, as pointed out by Healey and colleagues (Healey, De Ruiter, \& Mills, 2018), the study of miscommunication is a promising avenue for future research.

Before we discuss the general relevance of the line of research from which the current study originates, we note that this study serendipitously acquired specific relevance in light of a recent independent test of the Faithfulness Assumption (Micklos et al., 2020). To perform the test, Micklos and colleagues assigned a well-known referential communication task to a pair of participants. One participant (the director) communicated to the other (the matcher) which image to pick from a set of images (Clark \& Wiles-Gibbs, 1986; Krauss \& Weinheimer, 1964). Since the images had no pre-established names, participants had to perform a number of accurate communicative exchanges to identify the correct image. These exchanges were performed through instant messaging conversations. Micklos and colleagues found that the matchers' mean confidence in having succeeded at the referential communication task was higher for successful trials than for unsuccessful ones. Furthermore, an analysis of the instant messaging conversations revealed that, when matchers signaled communication problems through repairs, the pair was less successful at the task and the directors were correspondingly less confident. From these results, Micklos and colleagues concluded that "interlocutors exhibit (a degree of) sensitivity to problems in communication, and use repairs to signal and address them" (Micklos et al., 2020, p. 14). We agree with the conclusion, which is consistent with cross-linguistic observations (Dingemanse et al., 2015). However, we believe that a thoroughgoing assessment of the Faithfulness Assumption entails two questions that are more stringent: do people notice every communication problem they encounter and, when they do, do they systematically initiate a repair? Considering that in the current study we systematically observed whether people repaired a communication problem, the study provides an ideal opportunity to answer the second question. The answer is negative: People do not repair all of the communication problems they encounter.

As for the general relevance of the line of research from which the current study originates, we begin by noting that the existence of informational exchanges that violate the Faithfulness Assumption is consistent with two well-known facts.

First, it is consistent with the fact that miscommunication is rather common in daily life (Mustajoki et al., 2018; Tzanne, 2000). If people do not systematically repair the communication problems they encounter, or not even detect them, we should indeed expect miscommunication to be common. We note that this claim does not mean that people do not routinely perform faithful informational exchanges. If that were the case, in fact, we should expect communicational chaos, which is an expectation clearly inconsistent with the many achievements of human society. The claim simply means that sometimes people may not be fully aware of the requirements they need to meet to perform faithful exchanges, or may find them challenging. When the latter scenario happens, people may struggle to meet the requirements, or simply ignore them. Also, it is possible that non-informational interactions, which clearly fall outside of the Faithfulness Assumption's scope, are not only pervasive in 
ordinary circumstances but also tightly interwoven with informational exchanges. If this were true, it would imply substantial limitations to the scope of the Faithfulness Assumption.

This brings us to the second fact consistent with the existence of informational exchanges that violate the Faithfulness Assumption. When the faithfulness of the exchanges is of the utmost importance, people typically enforce the use of strict communication protocols. For example, this is the case for exchanges performed by armed personnel or by airplane pilots and control tower operators. If people were naturally predisposed to consistently meet the requirements of the Faithfulness Assumption, enforcing the use of strict protocols would be unnecessary. While these protocols likely serve a number of functions, we believe that a critical one among them is to counteract people's natural tendency to perform unfaithful exchanges.

The existence of informational exchanges that violate the Faithfulness Assumption is also consistent with the broad scientific claim that human cognition is designed to produce satisficing outcomes, not optimal ones (Simon, 1956). While this claim has been traditionally connected to the widespread existence of cognitive biases (Tversky \& Kahneman, 1974), recent research has documented the existence of similar communication biases (Keysar \& Henly, 2002; Keysar, Lin, \& Barr, 2003; Lane \& Ferreira, 2008; Savitsky, Keysar, Epley, Carter, \& Swanson, 2011). These biases suggest the hypothesis that human communication too might be designed to produce satisficing outcomes, not optimal ones (Ferreira \& Patson, 2007). We believe that our research on repair avoidance provides further evidence supporting this hypothesis. Because of methodological constraints, the evidence provided by the current study originates from strictly controlled conversational contexts. However, our previous studies suggest that converging evidence can be found in more natural conversational contexts (Galantucci et al., 2018; Roberts et al., 2016; Galantucci \& Roberts, 2014).

In conclusion, we would like to briefly speculate on the possibility that the research we presented here may offer new insights concerning the evolution of human communication. In particular, our studies suggest the hypothesis that human communication may have first evolved with low levels of faithfulness. Exchanges with low levels of faithfulness, in fact, are less taxing for our communicative abilities but can still provide enough benefits to be evolutionarily advantageous. The overall level of faithfulness is likely to have increased over time, particularly since the advent of writing, and this would be consistent with the intuitive appeal of the Faithfulness Assumption. However, when the human communicative abilities are assessed under proper conditions, we might still observe today the occurrence of exchanges that originate from the vestiges of a distant past. We believe that the hypothesis that human communication first evolved with low levels of faithfulness is complementary to the hypothesis that human communication first evolved as a form of "vocal grooming" (Dunbar, 1998) and hope that future research on people's present communicative abilities will explore this potential connection. 


\section{Author contributions}

B. Galantucci developed the study concept. All authors contributed to the study design. E. Spivack and N. Paley collected and processed the data, under the supervision of B. Galantucci and B. Langstein. B. Galantucci performed the data analysis and interpretation. B. Galantucci drafted the manuscript. All authors approved the final version of the manuscript for submission. We thank for helpful comments Mark Dingemanse, Samuel Dratch, Dov Fink, Carol Fowler, Simon Italiaander, Anthony Koutsoftas, Ariel Malka, Yosef Penner, Gareth Roberts, and all the students who helped us during various stages of the project.

\section{References}

Clark, H. H., \& Brennan, S. E. (1991). Grounding in communication. In L. B. Resnick, J. M. Levine, \& S. D. Teasley (Eds.), Perspectives on socially shared cognition (pp. 127-149). Washington, DC: American Psychological Association.

Clark, H. H., \& Wilkes-Gibbs, D. (1986). Referring as a collaborative process. Cognition, 22(1), 1-39.

Dingemanse, M., Roberts, S. G., Baranova, J., Blythe, J., Drew, P., Floyd, S., . . Manrique, E. (2015). Universal principles in the repair of communication problems. Plos One, 10(9), e0136100.

Dunbar, R. (1998). Grooming, gossip, and the evolution of language: Harvard University Press.

Fenn, K. M., Shintel, H., Atkins, A. S., Skipper, J. I., Bond, V. C., \& Nusbaum, H. C. (2011). When less is heard than meets the ear: Change deafness in a telephone conversation. Quarterly Journal of Experimental Psychology, 64(7), 1442-1456.

Ferreira, F., \& Patson, N. D. (2007). The 'good enough'approach to language comprehension. Language and Linguistics Compass, 1(1-2), 71-83.

Galantucci, B. (2005). An experimental study of the emergence of human communication systems. Cognitive Science, 29(5), 737-767. doi: 10.1207/s15516709cog0000_34

Galantucci, B. (2009). Experimental Semiotics: A New Approach for Studying Communication as a Form of Joint Action. Topics in Cognitive Science, 1(2), 393-410. doi: 10.1111/j.1756-8765.2009.01027.x

Galantucci, B., Fowler, C. A., \& Richardson, M. J. (2003). Experimental investigations of the emergence of communication procedures. In R. Sheena \& J. Effken (Eds.), Studies in Perception and Action VII Proceedings of the 12th International Conference on Perception E Action (ICPA) (pp. 120-124). Mahwah, NJ: Lawrence Erlbaum Associates.

Galantucci, B., \& Roberts, G. (2012). Experimental semiotics: An engine of discovery for understanding human communication. Advances in Complex Systems, 15(3-4). doi: 10.1142/S0219525911500263

Galantucci, B., \& Roberts, G. (2014). Do we notice when communication goes awry? an investigation of people's sensitivity to coherence in spontaneous conversation. Plos One, 9(7). doi: 10.1371/journal.pone.0103182

Galantucci, B., Roberts, G., \& Langstein, B. (2018). Content deafness: When coherent talk just doesn't matter. Language \& Communication. doi: 10.1016/j.langcom.2018.01.001

Galantucci, B., \& Steels, L. (2008). The emergence of embodied communication in artificial agents and humans. In I. Wachsmuth, M. Lenzen, \& G. Knoblich (Eds.), Embodied Communication in Humans and Machines (pp. 229-256). Oxford: Oxford University Press.

Goffman, E. (1955). On face-work: An analysis of ritual elements in social interaction. Psychiatry, 18(3), 213-231.

Goldin-Meadow, S., \& Feldman, H. (1977). Development of language-like communication without a language model. Science, 197(4301), 401-403. 
Golinkoff, R. M., Hirsh-Pasek, K., Bailey, L. M., \& Wenger, N. R. (1992). Young children and adults use lexical principles to learn new nouns. Developmental Psychology, 28(1), 99.

Healey, P. G., De Ruiter, J. P., \& Mills, G. J. (2018). Editors' Introduction: Miscommunication. Topics in Cognitive Science, 10(2), 264-278.

Hockett, C. F. (1960). Logical considerations in the study of animal communication. In W. Lanyon \& W. Tavolga (Eds.), Animal sounds and communications (pp. 392-430). Washington: American Institute of Biological Sciences.

Kegl, J., Senghas, A., \& Coppola, M. (1999). Creation through contact: Sign language emergence and sign language change in Nicaragua. In M. DeGraff (Ed.), Language Creation and Language Change: Creolization, Diachrony, and Development (pp. 179-237). Cambridge, MA: MIT Press.

Keysar, B. (2007). Communication and miscommunication: The role of egocentric processes. Intercultural Pragmatics, 4(1), 71-84. doi: 10.1515/ip.2007.004

Keysar, B., \& Henly, A. S. (2002). Speakers' overestimation of their effectiveness. Psychological Science, 13(3), 207-212.

Keysar, B., Lin, S. H., \& Barr, D. J. (2003). Limits on theory of mind use in adults. Cognition, 89(1), 25-41.

Krauss, R. M., \& Weinheimer, S. (1964). Changes in reference phrases as a function of frequency of usage in social interaction - A preliminary study. Psychonomic Science, 1(5), 113-114.

Lane, L. W., \& Ferreira, V. S. (2008). Speaker-external versus speaker-internal forces on utterance form: Do cognitive demands override threats to referential success? Journal of Experimental Psychology: Learning, Memory, and Cognition, 34(6), 1466.

Malinowski, B. (1923). The problem of meaning in primitive languages. In C. K. Ogden \& I. A. Richards (Eds.), The Meaning of Meaning (pp. 146-152). London: Routledge.

Micklos, A., Walker, B., \& Fay, N. (2020). Are People Sensitive to Problems in Communication?. Cognitive Science, 44(2), e12816.

Mustajoki, A., Sherstinova, T., \& Tuomarla, U. (2018). Types and functions of pseudo-dialogues. From Pragmatics to Dialogue, 31, 189.

Nisbett, R. E., \& Wilson, T. D. (1977). Telling more than we can know: Verbal reports on mental processes. Psychological Review, 84(3), 231.

Roberts, G., Langstein, B., \& Galantucci, B. (2016). (In)sensitivity to incoherence in human communication. Language \& Communication, 47, 15-22. doi: 10.1016/j.langcom.2015.11.001

Sandler, W., Meir, I., Padden, C., \& Aronoff, M. (2005). The emergence of grammar: systematic structure in a new language. PNAS, 102(7), 2661-2665. doi: 10.1073/pnas.0405448102

Savitsky, K., Keysar, B., Epley, N., Carter, T., \& Swanson, A. (2011). The closeness-communication bias: Increased egocentrism among friends versus strangers. Journal of Experimental Social Psychology, 47(1), 269-273.

Schegloff, E. A. (2000). When'others' initiate repair. Applied Linguistics, 21(2), 205-243.

Simon, H. A. (1956). Rational choice and the structure of the environment. Psychological Review, 63(2), 129.

Simons, D. J., \& Levin, D. T. (1998). Failure to detect changes to people during a real-world interaction. Psychonomic Bulletin \& Review, 5(4), 644-649. doi: 10.3758/bf03208840

Sperber, D., \& Wilson, D. (1986). Relevance: Communication and cognition. Cambridge (MA): Harvard University Press.

Tversky, A., \& Kahneman, D. (1974). Judgment under uncertainty: Heuristics and biases. Science, 185(4157), 1124-1131.

Tzanne, A. (2000). Talking at cross purposes: the dynamics of miscommunication. Amsterdam: John Benjamins. 
664 Clips A-D (footage from the study reported in Galantucci et al., 2018)

665

Clip A: Participant A

Clip B: Participant B

Clips 1-31 (footage from the current study)

Clip 1 (HPC condition): Participant 1

Clip 2 (HPC condition): Participant 2 (Because NP could not attend this session, there was a different confederate. It was again on of us, BL)

Clip 3 (HPC condition): Participant 3

Clip 4 (HPC condition): Participant 4

Clip 5 (HPC condition): Participant 5

Clip 6 (HPC condition): Participant 6

Clip 6a (HPC condition): Participant 6

Clip 7 (HPC condition): Participant 7 Example of repair

Clip 8 (LPC condition): Participant 8

Clip 9 (LPC condition): Participant 9

Clip 10 (LPC condition): Participant 10 (Because of a mistake, the confederate used a different coordinate for the instruction)

Clip 11 (LPC condition): Participant 11

Clip 12 (LPC condition): Participant 12

Clip 13 (LPC condition): Participant 13
Clip C: Participant C

Clip D: Participant D $\underline{\text { Clip } 14 \text { (LPC condition): Participant } 14}$

Clip 15 (LPC condition): Participant 15

Clip 16 (LPC condition): Participant 16

Clip 17 (LPC condition): Participant 17

Clip 18 (HPC condition): Participant 18

Clip 19 (HPC condition): Participant 19

Clip 20 (HPC condition): Participant 20

Clip 21 (HPC condition): Participant 21

Clip 22 (HPC condition): Participant 22 -

Example of repair

Clip 23 (LPC condition): Participant 23

Clip 24 (LPC condition): Participant 24

Clip 25 (LPC condition): Participant 25

Clip 26 (LPC condition): Participant 26

Clip 27 (LPC condition): Participant 27

Clip 28 (LPC condition): Participant 28

Clip 29(LPC condition): Participant 29

Clip 30 (LPC condition): Participant 30

Clip 31(LPC condition): Participant 31 